\title{
Chronic Candidal Bronchitis: A Consecutive Series
}

\author{
Douglas C. Johnson ${ }^{*}$ \\ Spaulding Hospital Cambridge, Cambridge, MA 02138, USA
}

Open Access

\begin{abstract}
Background: Persistent Candida from fungal cultures of respiratory secretions are often ignored and not treated due to assumptions concerning benign colonization.

Objectives: To determine the clinical course of patients with chronic sputum and fungi on culture, including response to antifungal treatment.
\end{abstract}

Methods: All patients seen at a single long term acute care hospital (LTAC) between May 2009 and September 2010 with at least two months of daily sputum and fungus on more than one sputum culture were identified. LTAC, inpatient, and outpatient records through June 2011 were reviewed to assess clinical features and response to therapy or to cessation of therapy.

Results: Eleven patients were identified, having sputum duration of 5 months to 28 years, and respiratory cultures growing Candida species. Fungi included C albicans (8 patients), C glabrata (2), C krusei (2), C tropicalis (1), C parapsilosis (1), Aspergillus fumigatus (1), Aspergillus terreus (1), and Scedosporium (1), the latter 3 in conjunction with Candida species. All had abnormal chest CT scans, often with bronchiectasis and sometimes atelectasis or consolidation, and ten of 11 patients were on chronic steroids (inhaled and/or systemic). Antifungal therapy, mostly oral voriconazole and nebulized amphotericin, led to improved respiratory symptoms and sputum within 3 weeks in 10 of 10 treated patients. Lack of antifungal therapy or early cessation of treatment was associated with progressive or recurrent symptoms and death of one patient.

Conclusions: This case series suggests that chronic candidal bronchitis is associated with significant morbidity and responds well to treatment. Such patients may benefit from extended antifungal therapy. Guidelines for the treatment of Candida in pulmonary secretions should be reevaluated.

Keywords: Antifungal, amphotericin, bronchiectasis, Candida, chronic bronchitis, prednisone, voriconazole

\section{INTRODUCTION}

Antibiotics are standard care for patients with chronic sputum who have bacteria on sputum culture, yet antifungal agents are often not used for patients with chronic sputum who have fungi on sputum culture. Antifungal therapy is standard treatment for Candida cultured from nearly all parts of the body, yet guidelines recommend not treating Candida from respiratory secretions, since such growth "rarely indicates invasive candidiasis" [1]. The author could not find any reports in the literature or any infectious disease or pulmonary society recommendations to treat Candida in patients with chronic bronchitis or mucous plugging.

In the author's over three decades experience, empiric antibiotic use is common for patients with bronchitis and no bacteria on culture, and antifungal therapy is very rare for patients with bronchitis and fungi on sputum culture.

This study addresses the question "Should Candida on sputum culture of patients with chronic bronchitis be ignored?" This consecutive case series includes patients with months to years of daily sputum and sputum cultures with

*Address correspondence to this author at the Baystate Medical Center, 759 Chestnut Street, Springfield, MA 01199, USA; Tel: 413-794-0051;

E-mail: dougjohnsonmd@gmail.com fungus. Most patients had marked clinical improvement with antifungal treatment, but for several patients antifungal treatment was shortened or never given, with persistent or worsening symptoms. The findings of this case series are novel and highlight the need to reevaluate the treatment of Candidiasis in patients with chronic bronchitis.

\section{METHODS}

The author reviewed all his inpatient consultations at a long-term acute care hospital (LTAC) from 5/2009-9/2010, and selected all patients with at least two months of daily sputum and fungus on more than one sputum culture, with at least two weeks between cultures.

LTAC, inpatient, and outpatient records to June 2011 were reviewed. Data included clinical history, history of smoking or asthma, duration of daily sputum, pulmonary cultures, inhaled and systemic steroid therapy, chest $\mathrm{x}$-ray and CT, galactomannan levels, antibiotic and antifungal therapy, and clinical course. Institutional Review Board approval was obtained from Spaulding Rehabilitation Hospital (2011-P000289). Assessments to evaluate response to therapy or to cessation of therapy included changes in dyspnea, cough, sputum, chest exam (rhonchi/wheezing), chest x-ray, and need for oral steroids. Improvement was indicated by reduced or resolved dyspnea, cough, sputum, rhonchi, wheezing, need for steroids, and improved chest $\mathrm{x}$-ray. 


\section{RESULTS}

Of 413 pulmonary consultations, 11 patients $(2.7 \%)$ were identified with chronic daily sputum for over two months and fungus on repeated sputum cultures. The cases are summarized in Tables $\mathbf{1}$ and $\mathbf{2}$ with case reports as follows:

Case 1: 82 year-old with COPD, over six years daily sputum production, nearly all sputum cultures with moderate to abundant yeast of two types without bacteria other than normal flora, admitted to acute hospital for increased dyspnea and atelectasis. Two bronchoscopies cleared plugs and copious thick mucous and cultures grew moderate to abundant yeast of two types without bacteria. She received high dose steroids and antibiotics. At LTAC antibiotics were discontinued, and she received 16 days of voriconazole, nebulized amphotericin, and oral and nebulized acetylcysteine. Sputum grew C parapsilosis. CXR improved, dyspnea and sputum resolved, and 10 days into antifungal treatment prednisone was tapered to off. She was discharged on voriconazole and nebulized amphotericin/acetylcysteine. A few days later, she was admitted to acute hospital to evaluate hypoxia. Chest CT showed "The previously seen bilateral consolidations and associated volume loss is nearly completely resolved." "The amphotericin/mucomyst nebulizers and voriconazole were discontinued because suspicion for fungal infection causing shortness of breath was low per pulmonology," and she was discharged after a few days.

Thirty-four days off antifungal therapy she was hospitalized with severe dyspnea, CXR showing opacities, and sputum with abundant C. albicans, abundant C. glabrata, and normal flora. Over the following three weeks she received high dose steroids, daily IV antibiotics, and no antifungals. Bronchoscopy found thick yellow and milky secretions, abundant polys and yeast, cultures growing $\mathrm{C}$. albicans, C. glabrata, normal flora absent. She had intubation, tracheostomy, further bronchoscopies, made comfort-measures-only, and died shortly after.

Case 2: 50 year-old with pulmonary hypertension, frequent hospitalizations for exacerbations of bronchiectasis, and five years of daily thick sputum. Sputum often had MRSA and nearly always yeast: C. glabrata, C. albicans and yeast not C. albicans. At LTAC intravenous antibiotics were completed. He was then treated with nebulized vancomycin and voriconazole for six weeks with complete resolution of sputum and cough by 3 weeks. He had minimal sputum for six months. Sputum recurred eight months after discharge with MRSA and yeast. He received intermittent antibiotics but no further antifungal, with continued sputum over the subsequent 12 months.

Case 3: 31 year-old asthmatic with five years of daily sputum, frequent antibiotics, prolonged high dose prednisone, frequent hospitalizations, and a diagnosis of chronic purulent bronchitis. Cultures rarely had bacteria, 44 of 60 sputum cultures and three of seven bronchoscopy samples over four years grew fungus. Most cultures had C. albicans. At LTAC, she received 23 days of voriconazole and nebulized amphotericin with much improved sputum. She weaned off prednisone (from $40 \mathrm{mg}$ daily) and dyspnea

Table 1. Age, Sex, Pack Year, Asthma, Inhaled Steroids Duration, Prednisone Duration, Sputum Duration, Galactomannan Antigen Level, and Chest CT Findings

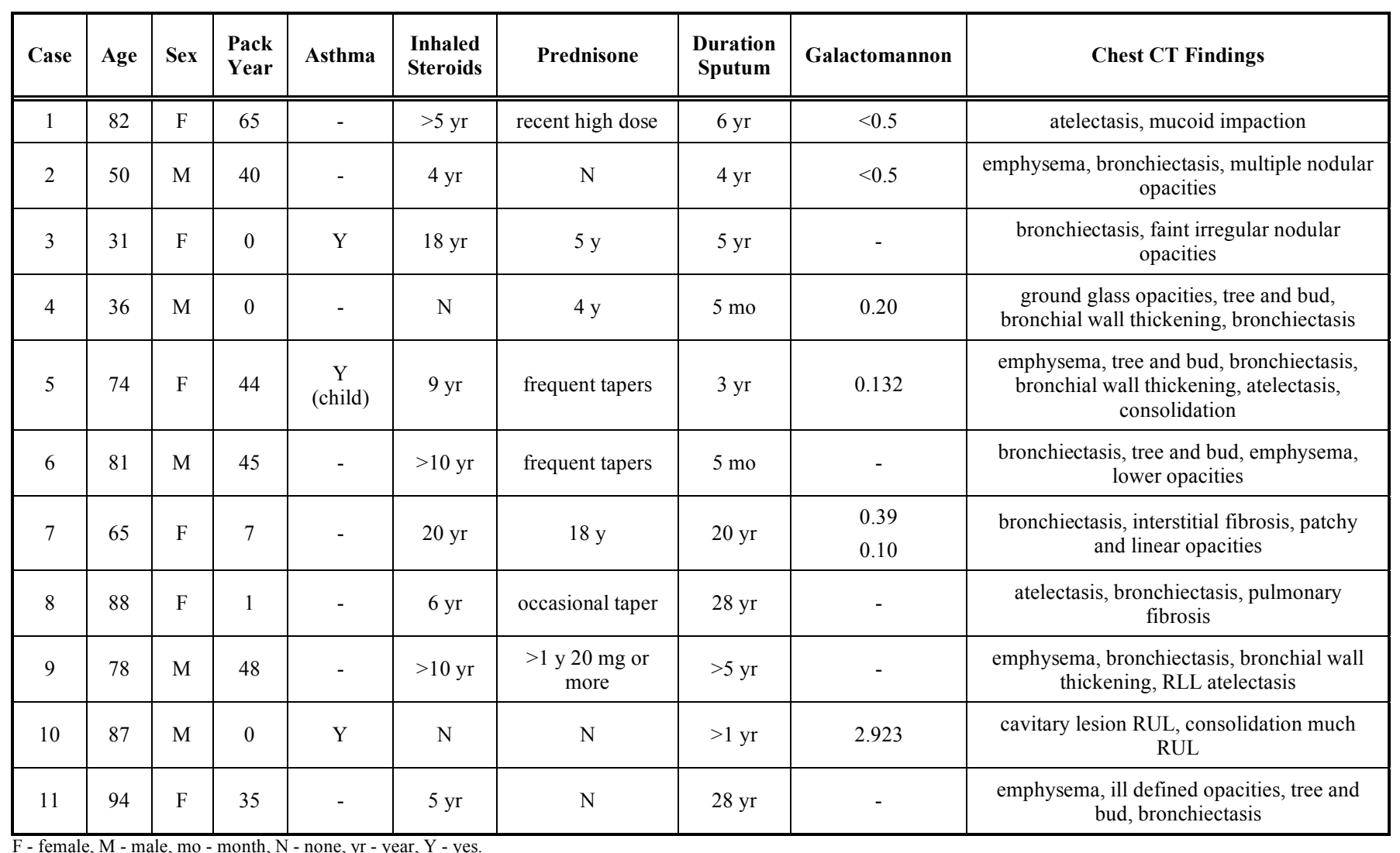


Table 2. Fungus, Antifungal Treatment, whether Tapered Off Prednisone, and Response

\begin{tabular}{|c|c|c|c|c|}
\hline Case & Fungus & $\begin{array}{c}\text { Antifungal } \\
\text { Treatment (Weeks) }\end{array}$ & Weaned Off Prednisone & Response \\
\hline 1 & C parapsilosis \& others & $2 \mathrm{~V} \& \mathrm{~A}$ & yes & $\begin{array}{l}\text { Resolution cough and sputum by } 2 \mathrm{wk} \text {, recurrence } 5 \mathrm{wk} \text { later, } \\
\text { then death } 3 \mathrm{wk} \text { later related to mucous plugging }\end{array}$ \\
\hline 2 & $\mathrm{C}$ albicans, $\mathrm{C}$ glabrata & $6 \mathrm{~V}$ & - & $\begin{array}{l}\text { Resolution of rhonchi and congested cough by } 3 \mathrm{wk} \text {, recurrence } \\
\text { at } 6 \text { mo }\end{array}$ \\
\hline 3 & $\mathrm{C}$ albicans \& others & $8 \mathrm{~V} \& \mathrm{~A}$ & yes & Improved sputum by $3 \mathrm{wk}$, with recurrence at $4 \mathrm{mo}$ \\
\hline 4 & C krusei & $16 \mathrm{~V}$ & prednisone for GVHD & $\begin{array}{l}\text { Resolution of rhonchi and congested cough by } 3 \mathrm{wk} \text {, continued } \\
\text { until death from multiorgan failure }\end{array}$ \\
\hline 5 & C tropicalis & $1 \mathrm{~V} \& \mathrm{~A}$ & $\begin{array}{l}\text { prednisone for } \\
\text { rheumatoid arthritis }\end{array}$ & $\begin{array}{l}\text { Resolution of cough and sputum by } 2 \mathrm{wk} \text {, with recurrence at } 5 \\
\text { mo }\end{array}$ \\
\hline 6 & $\mathrm{C}$ albicans, Aspergillus & $2 \mathrm{~V}$ & yes & Significant improvement by $2 \mathrm{wk}$, then recurrence $2 \mathrm{wk}$ later \\
\hline 7 & C albicans \& others & not treated & stayed on prednisone & Continued sputum and high prednisone over next year \\
\hline 8 & C albicans & $1 \mathrm{~F} \& \mathrm{~A}$, then $4 \mathrm{~F}$ & - & $\begin{array}{c}\text { Improved cough and sputum by } 2 \mathrm{wk} \text {, trauma then death } 3 \mathrm{mo} \\
\text { later }\end{array}$ \\
\hline 9 & C Krusei & $3 \mathrm{~F} \& \mathrm{~A}$, then $3 \mathrm{~V} \& \mathrm{~A}$ & yes & $\begin{array}{l}\text { Near resolution of cough, sputum, and hemoptysis after } 3 \mathrm{wk} \text { of } \\
\text { V\&A, died months later }\end{array}$ \\
\hline 10 & $\begin{array}{l}\text { C albicans, } \mathrm{C} \text { glabrata, } \\
\text { Aspergillus }\end{array}$ & $1 \mathrm{~V} \& \mathrm{~A}$ & - & Improved sputum over a week, then comfort measures and death \\
\hline 11 & $\mathrm{C}$ albicans \& others & $1.5 \mathrm{~V}$ & - & $\begin{array}{c}\text { Near resolution of cough, sputum by } 12 \text { days, with recurrence } \\
\text { after } 4 \text { mo }\end{array}$ \\
\hline
\end{tabular}

wk - weeks, mo - months.

Antifungal treatments include A - nebulized amphotericin, F - fluconazole, V - voriconazole. Voriconazole was generally 200 mg twice daily orally; nebulized amphotericin B 10 $\mathrm{mg}$ in $3 \mathrm{cc}$ sterile water daily or twice daily.

significantly improved. She took voriconazole and nebulized amphotericin for two months, with cultures showing no fungus one and three months following discharge. C. albicans was cultured from sputum starting two months after stopping antifungals. Twelve months following discharge she had copious sputum with normal flora and yeast and was on $40 \mathrm{mg}$ prednisone daily.

Case 4: 36 year-old with graft-versus-host disease post bone-marrow transplant, chronic immunosuppressive therapy, daily fluconazole, over six months of congested cough and sputum with cultures growing yeast, not identified. At LTAC, he was changed to voriconazole and given nebulized acetylcysteine. Sputum returned with $\mathrm{C}$ krusei. The sputum and cough fully resolved by three weeks. He stayed on voriconazole until his death four months later of multi-organ failure.

Case 5: 74 year-old with $\operatorname{IgA}$ deficiency on monthly intravenous immunoglobulin, chronic prednisone $7.5 \mathrm{mg}$ for rheumatoid arthritis, inhaled steroids, with thick sputum daily for three years. Sputum cultures included C. tropicalis (20), aspergillus fumigates (4), mycobacteria abscessus (2), and several with stenotrophomonas or pseudomonas. At LTAC, she received voriconazole, nebulized amphotericin and oral acetylcysteine for five days with significant improvement in sputum. At outpatient follow-up two weeks after discharge she "no longer felt infected" and antifungal treatment was stopped.

She was readmitted to LTAC 12 months later. She reported the sputum had nearly resolved a month after the first LTAC admission, until five months later when sputum and cough increased, with thick sputum daily since. At
LTAC, sputum had normal flora and C. tropicalis. Antifungals were given for five days, then stopped upon request of her outpatient pulmonary physician. Over the next months she continued with sputum, with cultures with $\mathrm{C}$. tropicalis, C. albicans, C. glabrata and pseudomonas.

Case 6: 81 year-old with frequent COPD exacerbations and hospitalizations, with over six months of daily sputum. Sputum culture had C. albicans, one colony Aspergillus, and varying bacteria. At LTAC, he received 16 days of voriconazole, with significant improvement in congestion and dyspnea, prednisone tapered to off, and was discharged with planned six week course of antifungal treatment. Ten days later he was readmitted to acute hospital not on antifungal with increased cough and dyspnea. Over the subsequent year he had continued sputum showing yeast with seven hospitalizations for increased dyspnea.

Case 7: 65 year-old with 20 years of daily sputum, on prednisone $20 \mathrm{mg}$ or higher for 18 years. C. albicans was cultured from most sputum and bronchoscopy samples over 17 years. Occasional sputum cultured unidentified yeast, rare Aspergillus, C. parapsilosis, or bacteria. At LTAC, antifungal treatment was recommended, but outpatient pulmonary physician would not agree to treatment, and prednisone could only be reduced to $50 \mathrm{mg}$ daily. Over the next 13 months she had continued daily sputum, was on prednisone $20 \mathrm{mg}$ or higher daily, and had C. albicans from all eight sputum and one bronchoalveolar-lavage culture.

Case 8: 88 year-old with 28 years of cough and sputum, on inhaled steroids and occasional prednisone taper, frequent hospitalizations for bronchoscopies to treat atelectasis, antibiotic courses, with bronchoscopy samples always with 
C. albicans and normal flora. At LTAC she was treated with fluconazole, nebulized and oral acetylcysteine for 8 days, with improvement in cough, shortness of breath, and sputum. Fluconazole was continued for 30 days as an outpatient. She did well until a fall three months later, sustaining rib fractures, then three days later was admitted to acute hospital with CXR showing likely atelectasis and pneumonia. Respiratory status worsened and she died.

Case 9: 78 year-old on chronic prednisone, frequent hospitalizations for COPD exacerbations, hypercapnia, with daily sputum for over five years showing unidentified yeast. At acute hospital he was treated with high dose steroids and antibiotics. At LTAC antibiotics, bronchodilators and steroids were continued, bilevel PAP given, and he developed hemoptysis. After three weeks at LTAC with continued daily two tablespoons of thick sputum, the author was asked for a second opinion. Sputum culture grew normal flora and C. albicans. Antibiotics were stopped and treatment was adjusted to include nebulized amphotericin B and oral fluconazole which he received for 21 days with some improvement. Sputum then grew C. krusei, antifungal changed to voriconazole with continued nebulized amphotericin. Over the next 18 days, sputum and hemoptysis nearly resolved, and prednisone tapered to off. He was discharged with plans to complete at least six weeks of voriconazole, and died several months later with respiratory problems.

Case 10: 87 year-old with over a year of sputum developed hemoptysis a few days after cholecystectomy. Bronchoscopy found secretions and blood from the right upper lobe and bronchial washings grew Aspergillus fumigatus, C. glabrata, and C. albicans. At LTAC he had continued sputum and cough. Antifungals were begun with significant improvement in sputum and cough after a week, and no further hemoptysis. He was started on a scopolamine patch, developed worse mental status, changed to comfort measures, antifungal therapy stopped, and IV morphine given for comfort. He died shortly thereafter.

Case 11: 94 year-old with 28 years of a few tablespoons thick daily sputum, several hospitalizations for worse sputum and dyspnea. Sputum cultures over six years had fungus on all 26 samples sent for fungal culture: C. albicans (26), C. glabrata (13), Scedosporium apiospermum (14), aspergillus fumigatus (3), yeast not C. albicans (2), penicillium (1), dematiaceous mold (1), Scedosporium prolificans (1). Mycobacterium avium complex on three samples, and some sputum with bacteria. She received frequent antibiotics and a course of anti-tuberculous therapy. At LTAC she received 11 days of voriconazole, with significant improvement in her sputum. Antifungal treatment was stopped on discharge by her outpatient pulmonary physician, who reported she had minimal cough a few days after discharge, and that on a visit three months after discharge "From a respiratory standpoint, she feels the best she has in months." Four months after discharge she was hospitalized with bronchiectasis exacerbation.

She was readmitted to the acute hospital 18 months after the first LTAC admission for worse sputum and dyspnea, and was treated with antibiotics. Of eight sputum cultures between the first and second LTAC admissions, all had yeast, a few had bacteria, and one mycobacterium avium complex. Upon transfer to LTAC she had a few tablespoons of sputum daily. Antifungal treatment was recommended, but her outpatient pulmonary physician objected to antifungals, so none were given. She continued with sputum and dyspnea.

\section{SUMMARY OF CASES}

All 11 patients had sputum duration for over five months, nine over a year, six over five years, and two over 20 years. Nine were on chronic inhaled steroids, four on chronic prednisone and four on intermittent prednisone. Fungus was present on most sputum cultures for prolonged periods. The primary fungus included C. albicans (5), C. krusei (2), C. tropicalis (1), while the other three patients had C. albicans with other fungi. All had abnormal chest CT scans, most with bronchiectasis and some with atelectasis or consolidation. The only patient with elevated $(>0.5)$ galactomannon levels also had aspergillus.

All 11 patients had persistent sputum despite antibiotic treatment at the acute hospitals prior to LTAC admission, and most had completed antibiotics prior to starting antifungals. Ten of the ten patients treated with antifungals had a good to excellent clinical response, with three having total resolution of sputum, and one having negative fungal cultures for a few months on the small amount of persisting sputum. All four on prednisone for pulmonary problems were weaned off prednisone over a few weeks. Only four patients completed six weeks of antifungal therapy. Sputum recurred in several patients, usually weeks or months following short courses of antifungal therapy. Five patients died within the follow-up period. One death was likely related to fungal mucous plugging in the setting of antibiotics, steroids, and no anti-fungals. Three others died with respiratory problems in which fungal bronchitis may have been a factor.

\section{DISCUSSION}

This case series suggests that candidal bronchitis is under treated. Just as patients suffer from chronic or recurrent bacterial bronchitis, pulmonary patients may have chronic or recurrent fungal bronchitis. This report suggests the importance of recognizing and treating chronic or persistent Candida in respiratory secretions. Lack of or incomplete antifungal treatments may lead to significant patient morbidity and mortality.

Most specialists follow guidelines recommending not treating fungus isolated from respiratory secretions. Current guidelines recommend treatment of Candida for 13 of 14 clinical conditions [1]. However, they do not recognize Candidal bronchitis, recommending that "Growth of Candida from respiratory secretions rarely indicates invasive candidiasis and should not be treated with antifungal therapy." Reasons cited for not treating fungi in sputum include "In contrast to pneumonia, colonization of the airway with Candida species and/or contamination of the respiratory secretions with oropharyngeal material are extremely common." However, as this series suggests, Candida respiratory secretions may indicate fungal bronchitis, not colonization, and can be associated with significant morbidity and sometimes mortality. Fungal bronchitis should be considered in patients with chronic 
sputum production or mucous plugging, especially in patients whose symptoms and findings do not resolve with antibiotic therapy and have fungi on sputum smear or culture.

The spectrum of pulmonary fungal infection is wide. While invasive fungal infection and aspergilloma are recognized, chronic bronchitis and mucous plugging related to fungal infections are not. Most studies on pulmonary fungal infection ignore fungi isolated from sputum, requiring fungus from tissue or pleural fluid culture [2]. There is some recognition that fungi in sputum has adverse health effects. Aspergillus tracheobronchitis describes aspergillus in sputum with thick mucous plugs [3]. C. albicans cultured from sputum of cystic fibrosis patients is associated with increased hospital-treated exacerbations and decline in FEV1[4].

Colonization is defined as the presence of an organism in the absence of symptoms or signs of infection. Patients with cystic fibrosis and long-term antibiotics, diabetes, and prolonged glucocorticoid use have increased colonization with C. albicans and other fungi [5,6]. Widespread use of inhaled steroids, systemic steroids, and antibiotics likely contribute to more frequent fungal colonization and fungal bronchitis.

Many patients had prior bacterial bronchitis or pneumonia. Fungal bronchitis may be a risk factor for bacterial infection. C. albicans is a risk factor for pseudomonas pneumonia in the intensive care unit [7]. Interactions between fungi and bacteria include $\mathrm{C}$. albicans affecting motility of Pseudomonas and transition between virulent and nonvirulent states [8], and Pseudomonas killing C. albicans hyphae but not yeast [9].

Patients with fungal bronchitis benefit from antifungal therapy. There was some improvement in sputum by one week and marked improvement by three weeks into therapy. However, symptoms and sputum generally recurred a few weeks to a few months after two to three weeks of therapy, and sometimes recurred a few to several months following six weeks of therapy. Patients with C. krusei improved with voriconazole but not fluconazole.

The optimal duration and mode of antifungal treatment is uncertain, as is the role of including nebulized and/or oral acetylcysteine. For patients with several months of fungal bronchitis, six weeks of therapy with combined oral antifungal and nebulized amphotericin was generally effective. It is likely a shorter duration of treatment would be needed for shorter duration bronchitis. If patients develop recurrent fungal bronchitis, treatment should be resumed. Further studies are needed to determine the effectiveness of nebulized amphotericin versus oral azole antifungal therapy alone and also to assess effective duration of antifungal therapy.

In conclusion, Candida and fungal growth in respiratory secretions can be associated with chronic sputum production, mucous plugging, bronchiectasis, and atelectasis, leading to chronic steroid use and recurrent hospitalizations. Fungal bronchitis is typically ignored, and may result in significant morbidity and sometimes mortality. Antifungal therapy for Candidal bronchitis led to dramatic improvement in patient symptoms and clinical status, while early cessation of therapy led to recurrence. Controlled clinical trials are needed to determine whether treatment guidelines should be modified to encourage effective identification and antifungal therapy for such patients.

\section{CONFLICT OF INTEREST}

The authors confirm that this article content has no conflicts of interest.

\section{ACKNOWLEDGEMENTS}

Spaulding Hospital Cambridge respiratory therapists provided nebulized amphotericin and acetylcysteine treatments. There was no funding for this study. The writing of this study was completed while the author was employed by Baystate Medical Center, Springfield, MA 01199, USA.

\section{REFERENCES}

[1] Pappas PG, Kauffman CA, Andes D, et al. Clinical practice guidelines for the management of Candidiasis: 2009 update by the Infectious Diseases Society of America. Clin Infect Dis 2009; 48: 503-35.

[2] Chen K, Ko SC, Hsueh PR, Luh KT, Yang PC. Pulmonary fungal infection: emphasis on microbiological spectra, patient outcome, and prognostic factors . Chest 2001; 120: 177-84.

[3] Denning DW. Commentary: unusual manifestations of aspergillosis. Thorax 1995; 50: 812-3.

[4] Chotirmall SH, O’Donaghue E, Bennett K, Gunaratnam C, O’Neill SJ, McElvaney NG. Sputum Candida albicans presages FEV1 decline and hospital-treated exacerbations in cystic fibrosis. Chest 2010; 138: 1186-95.

[5] Bakare N, Rickerts V, Bargon J, Just-Nübling G. Prevalence of Aspergillus fumigatus and other fungal species in the sputum of adult patients with cystic fibrosis. Mycoses 2003; 46: 19-23.

[6] Blaschke-Hellmessen R, Spitzer H, Paul KD, Hoffmann C. Mycological surveillance of children with cystic fibrosis. Mycoses 1991; 34: 43-7.

[7] Nseir S, Jozefowicz E, Cavestri B, et al. Impact of antifungal treatment on Candida-Pseudomonas interaction: a preliminary retrospective case-control study. Intensive Care Med 2007; 33: 137-42.

[8] Douglas LJ. Medical importance of biofilms in Candida infections. Rev Iberoam Micol 2002; 19: 139-43.

[9] Hogan DA, Kolter R. Pseudomonas-Candida interactions: an ecological role for virulence factors. Science 2002; 296: 2229-32. 